Article | Special Issue

\title{
How Mobility Technologies Change Our Lived Experiences: A Phenomenological Approach to the Sense of Agency in the Autonomous Vehicle ${ }^{1}$
}

Taehee Kim

\begin{abstract}
In mobility studies, it is commonly recognized that the development of mobility technologies brings about a vast range of changes in human lived experiences. Taking the autonomous vehicle as an example, this paper investigates the possible changes in human lived experiences resulting from said development. Based on phenomenological and cognitive scientific studies, this paper gives a detailed analysis of how the kinesthesis and sense of agency vary from everyday bodily movement to manual driving to autonomous driving following six levels of driving automation. This paper identifies, through philosophical analyses and interpretation, the problem of how the human driver can attend to driving without being fully engaged in it and suggests the possibility of considering the "driver-car" assemblage, a unified agent that gives relevant secondary attention to and is responsible for the driving situation. In doing so, this paper contributes to the discussions not only on the descriptive features of human lived experience but also on the normative issues around human drivers' responsibility in an autonomous vehicle.
\end{abstract}

Keywords: mobility, mobility technology, autonomous vehicle, kinesthesis

1 This work was supported by the Ministry of Education of the Republic of Korea and the National Research Foundation of Korea (NRF-2018S1A6A3A03043497).

(C) 2021 Taehee Kim

https://www.kritike.org/journal/special issue 2021/kim april2021.pdf

ISSN 1908-7330 


\section{MOBILITY TECHNOLOGIES}

\section{Introduction}

7 mobilities lens is considered a useful theoretical tool for analyzing modern societies where mobilities have increased dramatically due to various mobility technologies' developments. From the outset, the "new mobilities paradigm" in social sciences and humanities was meant to examine modern societies using this lens. Following Georg Simmel, who "sets an agenda that connects mobilities and materialities," focus has been put on the "hybrid systems, 'materialities and mobilities,' that combine objects, technologies, and socialities." 2 One of the most dramatic and well-known examples of mobility technology bringing about a vast range of changes in human lived experiences was rail travel in the nineteenth century. More recently, mobility technologies such as GPS navigation have notable effects on the lived experiences of travelers.

Still, "the prevailing phenomenon" is to operate with concepts "usually referring to technologies, not to people" and adopts a "view from above," rather than a "view from on the ground." 3 This phenomenon has given rise to the general ignorance of the lived experiences of "feelings for, of, and within cars," which are "socially and culturally embedded" in embodied sensibilities, practices, and cultures "that form around particular systems of automobility and generate different aesthetic and kinesthetic dispositions toward driving." 4 Therefore, one of my concerns here is to pay particular attention to the mutually influential relationship between the technological conditions for mobilities and human beings who develop and utilize such mobility technologies.

As a humanistic inquiry on mobilities, which is concerned, by nature, with how they are experienced and constituted by and with the meanings and values they represent to hypermobile modern societies and individuals, this paper is supposed to explore "the represented, imagined, and speculated dimensions" of mobilities, for instance, "by employing cultural-political, literary, and philosophical analysis and interpretation." 5 Among those characteristically humanistic approaches, this paper assumes the phenomenological perspective to consider "the most psychologically relevant aspects of the driving situation, namely the experiences of the

\footnotetext{
${ }^{2}$ Mimi Sheller and John Urry, "The New Mobilities Paradigm," in Environment and Planning A, 38 (2006), 214-215.

${ }^{3}$ Monika Büscher, Paul Coulton, Christos Efstratiou, Hans Gellersen, and Drew Hemment, "Connected, Computed, Collective: Smart Mobilities," in Mobilities: New Perspectives on Transport and Society, ed. by Margaret Grieco and John Urry (London: Routledge, 2015), 135.

${ }^{4}$ Mimi Sheller, "Automotive Emotions: Feeling the Car," in Theory, Culture \& Society, 21 (2004), 222.

${ }^{5}$ Jooyoung Kim, Taehee Kim, Jinhyoung Lee, and Inseop Shin, "Exploring Humanistic Layers of Urban Travel: Representation, Imagination, and Speculation," in Transfers, 9 (2019), 99.

(c) 2021 Taehee Kim

https://www.kritike.org/journal/special issue 2021/kim april2021.pdf

ISSN 1908-7330
}

(c) $)$ BY-NC-ND 
driver" that "traditional, quantitative data arguably fail to grasp." 6 The case of the autonomous vehicle, "a topical example of a socially embedded and potentially ubiquitous AI technology,"7 is taken as the subject of the inquiry since it appears to be of great significance for future mobilities.

Many discussions have been made about autonomous vehicles, mainly regarding their technological developments and their significances in broader social contexts. About the latter, a particular focus has been laid on regulating autonomous vehicles, especially from a legal and ethical perspective, since if the driver's "responsibilities, rights and authority that go with taking control of a vehicle would be denied," "the moral status of the driver" would be removed. ${ }^{8}$ In the current discussion, this problem is referred to mainly regarding the responsibility for harms and damages an accident may cause; issues of whom to blame among all the agents' involved in operating the vehicle, for instance, the car manufacturer, the human driver, the self-driving system on the car, or the transport planner. In those discussions, the philosophical, mostly ethical reasoning has mainly concentrated on applying, for instance, the trolley problem to the autonomous vehicle. In this regard, it is barely remembered that these normative issues concerning legal and ethical responsibility are inseparably linked to and, thus, supposed to be preceded by descriptive issues on the action of agents operating autonomous vehicles. This paper refers to one of these issues: the driver's sense of agency, in short, the agent's awareness of initiating, executing, and controlling voluntary action within autonomous vehicles. This, in turn, will play a substantial role in attributing the responsibility relevant to legal and ethical issues. ${ }^{9}$

\section{Driving Agent on an Autonomous Vehicle}

An autonomous vehicle, also known as a self-driving car or a driverless car, is a vehicle that drives itself autonomously without a driver; it is able "to operate without human intervention for extended periods of time

${ }^{6}$ Kjell Ivar Øvergård, "A Video-Based Phenomenological Method for Evaluation of Driving Experience in Staged or Simulated Environments," in Embodied Minds - Technical Environments: Conceptual Tools for Analysis, Design and Training, ed. by Thomas Hoff and Cato Alexander Bjørkli (Trondheim: Tapir Academic Press, 2008).

7 Martin Cunneen, Martin Mullins, and Finbarr Murphy, "Autonomous Vehicles and Embedded Artificial Intelligence: The Challenges of Framing Machine Driving Decisions," in Applied Artificial Intelligence, 33 (2019), 707.

${ }^{8}$ Eric Laurier and Tim Dant, "What We Do Whilst Driving: Towards the Driverless Car," in Mobilities: New Perspectives on Transport and Society, ed. by Margaret Grieco and John Urry (London: Routledge, 2015), 240.

${ }^{9}$ Patrick Haggard and Manos Tsakiris, "The Experience of Agency: Feelings, Judgments, and Responsibility," in Current Directions in Psychological Science, 18 (2009).

(C) 2021 Taehee Kim

https://www.kritike.org/journal/special issue 2021/kim april2021.pdf

ISSN 1908-7330

(cc) BY-NC-ND 


\section{MOBILITY TECHNOLOGIES}

and to perform a broad range of actions," 10 equipped with "a complex system that necessarily involves sensors, analytics, actuators and decision making elements, in which the default setting means humans are not directly involved in decision making."11 Especially with the convergence of information and communication technologies and intelligent transport systems embedded into autonomous vehicles, "an epochal shift" of the automobility system being "reconstituted as a network system rather than as separate 'iron cages,' as a potentially integrated nexus rather than as a parallel series" is imminent. ${ }^{12}$ The introduction of autonomous vehicles has further potential to impact developing carsharing systems at the heart of new mobility concepts and changing public transportation. ${ }^{13}$

However, the question of who the driving agent of the autonomous vehicle is remains more complicated and significant than it seems at first glance. At the level of "full automation" or "high automation," which will be discussed later, far from "driving" in the sense of freely performed human action, driving in which a human agent is by any means involved is unnecessary. Thus, the problem of the sense of agency during driving as an action freely performed by or by any means relevant to a human agent can be only at issue at the lower levels of driving automation on which this paper intends to focus.

Meanwhile, a human driver responsible for driving is the person using and controlling the vehicle and, through his/her sense of agency, the person being aware of his/her control. The nature and degree of this responsibility for a driving situation vary, depending on the various driving automation levels. Hence, to deal with these descriptive features of agency in the context of autonomous vehicles, the role of a human driver/occupant at each level is to be addressed. In the standard classification system published by the Society of Automotive Engineers International, six levels of driving automation ranging from "no automation" to "full automation" are identified: ${ }^{14}$

10 Patrick Lin, "Why Ethics Matters for Autonomous Cars," in Autonomous Driving: Technical, Legal and Social Aspects, ed. by Markus Maurer, Chris Gerdes, Barbara Lenz, and Hermann Winner (Cham: Springer Nature, 2016), 69.

11 Adam Henschke, "Trust and Resilient Autonomous Driving Systems," in Ethics and Information Technology, 22 (2020).

${ }^{12}$ John Urry, Mobilities (Cambridge: Polity Press, 2007), 134.

${ }^{13}$ Barbara Lenz and Eva Fraedrich, "New Mobility Concepts and Autonomous Driving: The Potential for Change," in Autonomous Driving: Technical, Legal and Social Aspects, ed. by Markus Maurer, Chris Gerdes, Barbara Lenz, and Hermann Winner (Cham: Springer Nature, 2016), 69.

${ }^{14}$ SAE International, "SAE International Releases Updated Visual Chart for Its 'Levels of Driving Automation' Standard for Self-Driving Vehicles," in SAE.org, (11 December 2018), $<$ https://www.sae.org/news/press-room/2018/12/sae-international-releases-updated-visual-

(C) 2021 Taehee Kim

https://www.kritike.org/journal/special issue 2021/kim april2021.pdf

ISSN 1908-7330

(cc) BY-NC-ND 
Level 0 (No Automation): The vehicle system performs only auxiliary functions such as emergency notification. The human driver performs all driving tasks such as steering and accelerating/decelerating, taking full responsibility for all aspects of varying driving situations.

Level 1 (Driver Assistance): An advanced driver assistance system (ADAS) can assist the human driver with either steering or accelerating/decelerating, but not both simultaneously. The human driver still handles all steering and accelerating/decelerating while monitoring the surrounding environment, taking full responsibility for all aspects of varying driving situations.

Level 2 (Partial Automation): An ADAS on the vehicle can control both steering and accelerating/decelerating simultaneously under some circumstances. The human driver is allowed to be disengaged from some tasks but must continue to pay full attention to monitoring the driving situation to take control of the vehicle back, if needed immediately. The human driver still takes full responsibility for most safety-critical functions and all monitoring of the environment.

Level 3 (Conditional Automation): An automated driving system (ADS) on the vehicle can itself perform all aspects of the driving task under some circumstances and is responsible for driving control and detecting variables while driving. While, up to Level 2, the system assists in some of the driving tasks, the system performs, from Level 3, entire driving tasks under some circumstances. For instance, the car can change lanes on its own, overtake the vehicle ahead, or avoid obstacles. The human driver must be ready to take control back, if needed, but does not need to continually monitor the driving situation, in contrast to Level 2. The driver's attention is still critical but can disengage from "safety-critical" functions like braking.

Level 4 (High Automation): An ADS on the vehicle can itself perform all driving tasks and monitor the driving situation under certain circumstances, and, contrary to Level 3, should itself respond safely even in a dangerous situation. The human driver need not pay attention to anything. However, in certain conditions, such as bad weather, driver intervention may be required, so a driving control device, such as a steering wheel, is necessary.

Level 5 (Full Automation): An ADS on the vehicle can do all the driving under all circumstances. The human driver turns to a passenger, ${ }^{15}$ never involved in driving except deciding the intended destination. This level of autonomous driving requires absolutely no human attention. Compared

chart-for-its- $\% \mathrm{E} 2 \% 80 \% 9$ Clevels-of-driving-automation $\% \mathrm{E} 2 \% 80 \% 9 \mathrm{D}$-standard-for-self-drivingvehicles>.

15 Tim Dant, "Drivers and Passengers," in The Routledge Handbook of Mobilities, ed. by Peter Adey, David Bissell, Kevin Hannam, Peter Merriman, and Mimi Sheller (London: Routledge: 2014), 368.

(C) 2021 Taehee Kim

https://www.kritike.org/journal/special issue 2021/kim april2021.pdf

ISSN 1908-7330

(cc) BY-NC-ND 


\section{MOBILITY TECHNOLOGIES}

to Level 4, the ADS at this level has no restrictions on areas where autonomous driving is possible. There is no need for pedals, brakes, or a steering wheel, as the ADS controls all critical tasks, monitoring the environment and identifying unique driving conditions like traffic jams.

At Levels 4 and 5, the vehicle is capable of all of driving tasks such as steering, braking, accelerating, monitoring as well as responding to events, determining when to change lanes, turn, and use signals, either under certain circumstances in the case of Level 4 or under all circumstances when Level 5 is reached. For that reason, although a full-fledged exploration of the driver's experience in an autonomous vehicle may require reviewing all of these levels, these levels, to which most discussions from the ethical and legal perspective are typically devoted, are not relevant to the state of affairs under current discussion: the sense of agency pertinent to the human driver's responsibility. In any case, these driving automation levels are technically challenging to materialize in a short period, and it also remains controversial whether materializing them is desirable at all.

Therefore, Levels 1 to 3 are most relevant for this paper. Among these levels, Level 3 is of particular interest, since, at this level, an awkward demand comes up; being allowed to be "disengaged" from most of the driving situation, the human driver must intermittently be "somehow engaged" in it to respond swiftly to changes and take control back at any time when the ADS requests to do so. However, prior to dealing with how this demand could be met, the sense of agency existent in two other cases will be briefly discussed, that is, in the case of bodily movement and of manual driving (Level 0) to identify how and to what extent human driver's sense of agency changes in the automated driving situation.

\section{Sense of Agency in the Case of Bodily Movements}

The concept of kinesthesis may hold some clues to elucidate the sense of agency in bodily movements. In this paragraph, this concept is explained not in driving situations but in everyday situations, then applied to driving situations. ${ }^{16}$ Kinesthesis is basically "the sixth sense that informs one what the body is doing in space through the sensations of movement registered in his joints, muscles, tendons and so on." 17 This concept is initially defined in this narrow sense as "the sensation of movement, particularly the sense of muscular effort relating to voluntary embodied movements." However, it can be extended beyond this narrow meaning "simply associated with the

\footnotetext{
${ }^{16}$ For a more detailed description of kinesthesis, see Taehee Kim, "Significance of Kinaesthesis for Mobility Humanities: A Phenomenological Contribution to the New Mobilities Paradigm," in Universitas-Monthly Review of Philosophy and Culture, 47 (2020).

${ }^{17}$ Urry, Mobilities, 48.

(c) 2021 Taehee Kim

https://www.kritike.org/journal/special issue 2021/kim april2021.pdf

ISSN 1908-7330
}

(c) $)$ BY-NC-ND 
muscular sensations of those who move" toward a broader spectrum, namely, "movement enacted, felt, perceived, expressed, metered, choreographed, appreciated and desired," which presumably has the potential of being "a common thread running through much arts and humanities research on mobility." 18

This concept, which was initially coined in physiology and used in physiology and psychology, was appropriated by Edmund Husserl, who imbued it with philosophical or phenomenological meaning. In Husserl's schema, it plays a significant role not only in the sensation of one's bodily movement but for the perception of external things. Husserl's discussion of kinesthesis draws on philosophical thought experiments about how visual perception of external three-dimensional objects is possible at all, even though, in a single moment, the object shows only a single aspect, a single adumbration (Abschattung) in the Husserlian term, from the singular perspective of the observer. At that moment, for instance, only (a part of) the facade of a house comes into the observer's sight. However, not only this single part but the entire object, including the side and rear, somehow come into the observer's awareness or are "perceived" in a broader sense. How is that possible?

The answer that Husserl proposes draws on the function of kinesthesis for perception. Although you see exclusively (a part of) an object, if you turn your eyes a little, then another part of the facade that was just on the periphery of your visual field or entirely outside your visual field now comes into the center of the visual field. Not only that, if you move around the house, then you can see the rear aspect of the house. Concerning this functional relation of if-then, the object's sensations (aesthesis) vary in an orderly manner, according to the observer's bodily movements (kines). Kinesthesis refers to the implicit knowledge of these functional relations between voluntary movements and an object's sensations, without which the "skillful coping" 19 with things is not at all conceivable.

This notion of kinesthesis is relevant to our discussion to the extent that such an implicit knowledge of these functional relations implies the freedom of action, as Husserl emphasizes: "At the same time, it follows that functions of spontaneity belong to every perception. The processes of the kinesthetic sensations are free here, and this freedom in the consciousness of

\footnotetext{
${ }_{18}$ Peter Merriman and Lynne Pearce, "Mobility and the Humanities," in Mobility and the Humanities, ed. by Peter Merriman and Lynne Pearce (London: Routledge, 2018).

${ }^{19}$ Hubert L. Dreyfus, Skillful Coping: Essays on the Phenomenology of Everyday Perception and Action, ed. by Mark Wrathall (Oxford: Oxford University Press, 2014).
}

(C) 2021 Taehee Kim

https://www.kritike.org/journal/special issue 2021/kim april2021.pdf

ISSN 1908-7330 


\section{MOBILITY TECHNOLOGIES}

their unfolding is an essential part of the constitution of spatiality." 20 This emphasis on the freedom to move that is by definition implied in kinesthesis means this notion plays an essential role in the discussion of agency. Experiencing your bodily movements, not merely as something that is happening to you (sense of ownership), but as something you are voluntarily doing (sense of agency), ${ }^{21}$ presupposes the kinesthesis; you have to move voluntarily and know the functional relations between these voluntary movements and the sensations caused by these movements. Given this kinesthesis, you know that you are the "author" of this action in most cases of action, even if you do not need to reflect on that explicitly. Given this kinesthesis, in most cases of action, you know that you are the "author" of this action, even if you do not need to reflect on that explicitly.

No matter how critical such analyses are, identifying the role of kinesthesis in creating the sense of agency is one thing; identifying how the sense of agency is structured is another. This sense of agency is indeed so intricate as to be "the product of several contributory elements: efferent signals, sensory (afferent) feedback, and intentional feedback, which is perceptual in nature." 22

Firstly, a sense of agency or "a phenomenal experience of agency" may be generated by efferent signals, "the signals the brain sends to the muscles to make them move." 23 That is because these signals at the subpersonal or unconscious level are, though in a complex non-linear manner, related to intentions and volitions to move oneself and to immediate anticipations, i.e., protentions in Husserl's terminology, of imminent bodily movements and their corresponding external results at the personal or conscious level.

Secondly, a sense of agency may also be produced by sensory feedback, "visual and proprioceptive/kinesthetic information that tells me that I'm moving," 24 that corresponds to the proprioception or kinesthesis in the above-mentioned narrow sense transmitted through muscle sensation or joint sensation. Sensory information transmitted from these sensations alone can indeed generate a sense of ownership, the awareness of one's moving body, but not a sense of agency because it can be transmitted even when a specific external force involuntarily moves one's body. Therefore, the sense

\footnotetext{
${ }^{20}$ Edmund Husserl, Ideas Pertaining to a Pure Phenomenology and to a Phenomenological Philosophy, Book II, trans. by R. Rojcewicz and A. Schuwer (Boston: Kluwer Academic Publishers, 1989), 63.

${ }^{21}$ Tim Bayne, "The Phenomenology of Agency," in Philosophy Compass, 3 (2008), 183.

22 Shaun Gallagher and Dan Zahavi, The Phenomenological Mind: An Introduction to Philosophy of Mind and Cognitive Science (New York: Routledge, 2008), 186.

${ }^{23}$ Ibid., 182.

${ }^{24}$ Ibid., 183.

(c) 2021 Taehee Kim

https://www.kritike.org/journal/special issue 2021/kim april2021.pdf

ISSN 1908-7330
}

(c) $)$ BY-NC-ND 
of agency is possible only when such information counts truly as feedback from efferent signals related to the voluntary movements.

Thirdly, a sense of agency may be generated by intentional feedback, "some ... sense that my action is having an effect," ${ }^{25}$ both perceptual and practical. The former is the awareness that one's bodily movements motivate changes in perception, while the latter is the awareness that one's bodily movements motivate changes in things in themselves, such as their displacement or deformation.

The sense of agency issued by voluntary bodily actions involves efferent signals, afferent feedback, and intentional (perceptual and practical) feedback. ${ }^{26}$ However, their precise relations discussed among neuroscientists, cognitive scientists, and phenomenologists are not pursued further in this paper. Instead, these insights will be used to account for the sense of agency felt in a driving situation.

\section{Sense of Agency in the Case of Manual Driving}

The first concern is to what extent the above-mentioned accounts for the sense of agency might be applied to the case of manual driving. The implicit knowledge of the functional relationship between our voluntary bodily movements and sensations enables us to cope with the world skillfully. Therefore, if we are skilled drivers, we experience the cars as extending our body or "dilating our being-in-the-world," as Maurice Merleau-Ponty puts it.

To get used to a hat, a car, a stick is to be transplanted into them, or conversely, to incorporate them into the bulk of our own body. Habit expresses our power of dilating our being-in-the-world or changing our existence by appropriating fresh instruments. ${ }^{27}$

In the same vein, Don Ihde's phenomenological insights suggest that the car becomes a symbiotic extension of the driver's body. ${ }^{28}$ In this "embodiment relation," human beings and technologies such as cars they are

${ }^{25} \mathrm{Ibid} ., 186$.

${ }^{26}$ These three elements of the sense of agency are closely related to the concept of kinesthesis construed by Husserl, consisting of the (self-)consciousness of the perceiver's bodily movements and the consciousness of external objects' perceptual changes. Namely, the former consciousness is caused by efferent signals and sensory feedback, while the latter by intentional feedback.

${ }^{27}$ Maurice Merleau-Ponty, Phenomenology of Perception (London: Routledge, 1962), 143.

${ }^{28}$ Don Ihde, "The Experience of Technology: Human-Machine Relations," in Cultural Hermeneutics, 2 (1974), 272.

(C) 2021 Taehee Kim

https://www.kritike.org/journal/special issue 2021/kim april2021.pdf

ISSN 1908-7330

$(\mathrm{Cc}) \overline{\mathrm{BY}-\mathrm{NC}-\mathrm{ND}}$ 


\section{MOBILITY TECHNOLOGIES}

riding form an embodied unity which, as a whole, is directed at the world. As we speak with other people through the phone, rather than speak to the phone itself, and as we look through a microscope rather than at it, the car is between the human and the external world, being a transparent means of experience. ${ }^{29}$

Experienced drivers have, in most driving cases, tacit know-how as the procedural knowledge, for instance, of the size and shape of their "extended body," namely, the car they are driving, so they can easily pass narrow alleyways and perform parallel parking in a small parking lot, without an enormous explicit and conscious effort. Don Ihde explains this skilled coping of drivers as follows:

One embodies the car, too, in such activities as parallel parking: when well embodied, one feels rather than sees the distance between car and curb - one's bodily sense is "extended" to the parameters of the driver-car "body." 30

Merleau-Ponty finds a direct analogy of this skill of experienced drivers to our everyday actions.

The driver assembles their learnt skill with the functionality of a car to be able to enter a narrow opening and see that I can "get through" without comparing the width of the opening with that of the wings, just as I go through a doorway without checking the width of the doorway against my body. ${ }^{31}$

In this context, the claim might be made that the driver-car has the same kind of implicit body-awareness or body schema (schéma corporel) ${ }^{32}$ as the one that enables us, in everyday actions, to recognize the affordances that surroundings exercise and to react appropriately to them. This body schema is a crucial element of the driver-car's kinesthesis. Owing to the "extended body schema," if you will, experienced drivers generally possess an

${ }^{29}$ Don Ihde, Technics and Praxis: A Philosophy of Technology (Dordrecht: Reidel, 1979), 8. For a concise summary of three possible relations between human beings and technology suggested by Ihde, see Benjiemen A. Labastin, "A Search for a Model of Critical Engagement with Technology: Feenberg's Instrumentalization Theory or MASIPAG's Struggle against Corporate Control of Agricultural Technologies?," in KRITIKE: An Online Journal of Philosophy, 13 (2019), 95.

${ }^{30}$ Don Ihde, Technology and the Lifeworld: From Garden to Earth (Bloomington: Indiana University Press, 1990), 74.

${ }^{31}$ Merleau-Ponty, Phenomenology of Perception, 143.

${ }^{32}$ Cf. Ibid., 98.

(C) 2021 Taehee Kim

https://www.kritike.org/journal/special issue 2021/kim april2021.pdf

ISSN 1908-7330

(c) BY-NC-ND 
"extended kinesthesis" or, as Nigel Thrift puts it, an "unnatural kinesthetics of the car ride" "in which the identity of the person and car kinesthetically intertwine," 33 so that they can act quickly and intuitively.

Thus, we can distinguish the kinesthesis of the driver and the extended kinesthesis of the driver-car. The drivers are aware of their bodily actions with the former kinesthesis, such as turning the steering wheel left or right, as well as the resulting perceptual and practical changes such as the car's turning left or right. Further, the driver-car is, with the latter kinesthesis, aware of its turning left or right and the resulting perceptual changes in the landscape surrounding it. In other words, the drivers possess two kinds of kinesthesis: a kinesthesis in a proper sense regarding the functional relations between their bodily actions and the changes in the car's state, as well as an extended or unnatural kinesthesis regarding the functional relations between the "actions" of the driver-car's extended body and the changes in the environment. Creating an analogy between the kinesthesis of everyday bodily actions and the extended kinesthesis of manual driving, the accounts of kinesthesis in manual driving can include the elements mentioned earlier: efferent signals, sensory feedback, and (perceptual and practical) intentional feedback.

Firstly, when drivers move their body for driving, for instance, when they step on the gas pedal or the brake pedal for longitudinal movements of the car or operate the steering wheel for lateral movements of it, efferent signals at the subpersonal level are transmitted from the motor area of the brain to the muscles. These signals are correlated to intentions and volitions to bring about specific actions that can be peculiarly conscious at the personal or phenomenological level. Novice drivers, in particular, are likely to have a higher degree of such conscious intentions and volitions so that they have to continuously give conscious efforts to come up with, for example, how much to turn the wheel along a curved road. By contrast, skilled drivers process this work nearly automatically so that the intentions and volitions are barely conscious. ${ }^{34}$

Secondly, these bodily movements then result in the movements of the steering wheel or of the gas/brake pedal, which in turn cause sensory afferent feedback, in other words, proprioceptions transmitted through muscle sensation or joint sensation. For both novice and experienced drivers, this feedback is usually transparent unless attention is required, for instance, if some sensory-motor incongruence is felt.

\footnotetext{
${ }^{33}$ Nigel Thrift, "Driving in the City," in Theory, Culture E Society, 21 (2004), 46-47 (as cited in Sheller, "Automotive Emotions," 228).

${ }^{34}$ This is well shown in the renowned case of the long-distance truck driver proposed by David M. Armstrong. See David M. Armstrong, "The Nature of Mind," in Arts: The Proceedings of the Sydney University Arts Association, 3 (1966), 46.
} 


\section{MOBILITY TECHNOLOGIES}

Thirdly, the drivers' bodily movements bring about some intentional feedback that can be classified in two ways: from where and for whom they are transmitted. ${ }^{35}$ Concerning the first way of classification, first of all, sensory feedback from (parts of) the vehicle can be called immediate: the visual appearance and haptic smoothness of a rotating steering wheel, the felt resistance of the gas and brake pedal, the sound of the engine, or changes in the sense of balance resulting from a sharp turn. Then, sensory feedback from the environment can be called mediate: the acoustic and haptic changes felt from the transformation of the roadbed or the visual changes in landscape resulting from the driver's movement.

Concerning the second mode of classification, feedback for novice drivers or experienced drivers is different in most cases. For instance, sensory feedback from the steering wheel must be quite apparent to novice drivers, who consistently make an effort to find out how much to turn the steering wheel when going around a sharp curve and flex their arms to turn the wheel that much. By contrast, experienced drivers usually are not that conscious of such feedback, since (parts of) the car feels literally like their (extended) body; it is analogous to the fact that we are, under normal circumstances, not conscious of our hand or mouth when drinking a cup of water with our hand and mouth. Still, experienced drivers are more likely to aware of the minute irregularities and abnormalities of their car or the familiar environment because their attentional resources are not exhausted by relentless efforts to deal with operating the vehicle itself. ${ }^{36}$

Having described the sense of agency based on the kinesthesis in the case of manual driving, that is, at Level 0 of driving automation, we can move, with this knowledge, to the case of autonomous driving.

\section{Sense of Agency in the Case of Autonomous Driving}

A sense of responsibility is given by being "vividly aware of being in control of our actions" resulting, "first, from intentional binding which creates a perception of agency, linking an intentional action to its outcome and, second, from the counterfactual reasoning that we could have chosen some other action." ${ }^{37}$ Thus, a sense of agency is not a sufficient, but at least a

${ }^{35}$ It is important to note that intentional feedback is feedback from the vehicle or environment grasped by the driver's intentionality, whereas sensory feedback is the feedback from within the driver's body.

${ }^{36}$ A real expert in driving would be defined, according to Dreyfus's model of skill acquisition, regarding four qualities: situational recollection, holistic recognition, intuitive decision, and absorbed awareness. See Stuart E. Dreyfus and Hubert L. Dreyfus, A Five-Stage Model of the Mental Activities Involved in Directed Skill Acquisition (Berkeley: California University Berkeley Operations Research Center, 1980), 15.

${ }^{37}$ Chris D. Frith, "Action, Agency and Responsibility," in Neuropsychologia, 55 (2014), 137.

(C) 2021 Taehee Kim

https://www.kritike.org/journal/special issue 2021/kim april2021.pdf

ISSN 1908-7330

(cc) BY-NC-ND 
necessary condition for feeling responsibility from a first-person perspective. Given this, and the fact that technological developments have "the potential to turn all drivers into passengers," 38 it would be strange for mere occupants of autonomous vehicles to have such a feeling.

The higher the level of driving automation is, the less the vehicle occupants engage in driving or, the less they can feel responsible for driving. At the final level of full automation, they need nothing but to have "intentionality of purpose," i.e., "the directing of consciousness to where the mobile subject is to go," without having "intentionality of progress," i.e., "the directing of consciousness to how mobility will be realized." Furthermore, "even the agency of the passenger to select their route may be restricted once intentionality of progress is managed on a collective rather than an individual basis" 39 in an intelligent transport network system. In this case, it would be difficult to define the scope of the individual responsibility, for an individual traffic participant can rarely have responsibility from the ethical point of view, if "you can want an outcome without wanting to produce that outcome" preferring "that the outcome merely materialize." 40

In this respect, the descriptive issues of how and to what extent drivers or occupants of an autonomous vehicle can have a sense of agency are closely connected to the normative issues of who could and should take responsibility for driving and, especially, for a traffic accident. Whereas the minimal precondition for an agent to take responsibility is that "the agent must have control over the action and know what she is doing," 41 it is the latter condition of knowledge that refers to the sense of agency we are discussing. Moreover, in that sense, it can be assumed that the sense of agency will gradually decrease as the level of driving automation gets higher from Level 0 to Level 5. Then, provided drivers lose a certain degree of their sense of agency in an autonomous vehicle, how far are they supposed to take responsibility, and how far are they relieved of their responsibility? Even at Levels 1 and 2, while drivers have almost the same basic sense of agency as at Level 0, such functions as a lane-keeping assist system or an automatic braking system can make the driver feel restricted from freely acting and, therefore, limit their sense of agency..$^{42}$ The transition from Level 2 to Level 3 is more dubious. At Level 3, ADS on the vehicle alone is responsible for driving performances, and drivers come to be allowed to take their hand off

\footnotetext{
${ }^{38}$ Dant, “Drivers and Passengers," 368.

${ }^{39}$ Ibid., 370-371.

${ }^{40}$ Bernard Williams, Ethics and the Limits of Philosophy (London: Routledge, 2011), 62.

${ }^{41}$ Mark Coeckelbergh, "Responsibility and the Moral Phenomenology of Using Selfdriving Cars," in Applied Artificial Intelligence, 30 (2016), 751.

42 Bruno Berberian, Jean-Christophe Sarrazin, Patrick Le Blaye, and Patrick Haggard, "Automation Technology and Sense of Control: A Window on Human Agency," in PLOS One, 7 (2012).
}

(C) 2021 Taehee Kim https://www.kritike.org/journal/special issue 2021/kim april2021.pdf ISSN 1908-7330 


\section{MOBILITY TECHNOLOGIES}

the steering wheel and be exempted from constant monitoring the driving situation. Still, if the system's requirements are exceeded, drivers are requested by the system to intervene. Until drivers take the control back, however, certain safety risks can arise. Some manufacturers even decided to skip Level 3 altogether to avoid these risks, ${ }^{43}$ while others are somewhat optimistic. ${ }^{44}$ It seems contradictory to let the driver's attention roam free, yet at the same time to request they pay enough attention to allow them to engage in a critical situation promptly. These issues are crucial because it could be considered excessively demanding for drivers who are not responsible at all for driving tasks to be unfailingly attentive and ready for a specific intervention; their sense of agency will likely have disappeared or at least decreased ${ }^{45}$ because, disengaged from the driving tasks, they do not possess any kinesthesis. This problem of the "irony of automation" results in "difficulties in maintaining an appropriate degree of situation and system awareness." 46

How could drivers, despite their lack of kinesthesis and their corresponding lack of sense of agency, still maintain situation and system awareness and the required kind or degree of attention to the driving situation which changes moment by moment? For drivers to maintain situational awareness (SA), their cognitive activities are to be situated "in the context of task-relevant inputs and outputs." 47 In other words, the situation relevant to these driving tasks does not refer to all the environmental factors surrounding the driver-car but the totality of task-relevant factors, among others. For a driver, for example, road conditions or traffic signal systems are elements of the situation in this sense, but road colors, pedestrians who do not try to cross the road, or pedestrian overpass, etc., are generally excluded from this situation. Still, the situation is continuously changing. In most cases, the other cars in front of the vehicle belong to the situation. In contrast, the cars behind the vehicle, except for the car immediately behind the vehicle representing the potential risk of a rear impact, are instantly excluded from the situational awareness because they are not relevant to driving tasks.

${ }^{43}$ Russ Mitchell, "When Robots and Humans Take Turns at the Wheel," in Los Angeles Times (22 September 2016), <https://www.latimes.com/business/autos/la-fi-hy-driverless-levelstesla-ford-gm-mercedes-volvo-google-20160922-snap-story.html>.

${ }^{44}$ Michael Martines, "Ford Rethinks Level 3 Autonomy," in Automotive News Europe (20 January 2019), <https://europe.autonews.com/automakers/ford-rethinks-level-3-autonomy>.

45 Wen, Yoshihiro Kuroki, and Hajime Asama, "The Sense of Agency in Driving Automation," in Frontiers in Psychology, 10 (2019), 9.

46 Ingo Wolf, "The Interaction Between Humans and Autonomous Agents," in Autonomous Driving: Technical, Legal and Social Aspects, ed. by Markus Maurer, Chris Gerdes, Barbara Lenz, and Hermann Winner (Cham: Springer Nature, 2016), 104.

47 Margaret Wilson, "Six Views of Embodied Cognition," in Psychonomic Bulletin $\mathcal{E}$ Review, 9 (2002), 626.

(c) 2021 Taehee Kim

https://www.kritike.org/journal/special issue 2021/kim april2021.pdf

ISSN 1908-7330

(cc) BY-NC-ND 
Based on this notion of the situation as the totality of task-relevant factors, three kinds of attention can be distinguished to tackle the problem of Level 3 that, while drivers are exempted from the duty to attend to driving, specific attention is still required to engage in a critical situation. In Aron Gurwitsch's phenomenological analyses on this sense of attention, the scopes to which the attention is directed are distinguished into the "theme," "thematic field," and "margin," 48 which correspond to the categories of Edmund Husserl's terminology, "primary attention," "the secondary attention relevant to the situation," and "the secondary attention irrelevant to the situation," 49 respectively.

The second kind of attention, i.e., the relevant secondary attention to the thematic field, can count as required of a driver riding the autonomous vehicle at Level 3. In other words, directing primary attention to some driving-irrelevant tasks, the driver must continuously pay secondary attention to the driving situation as a thematic field. A question that immediately arises is this: How is this possible without any real voluntary action for driving?

To answer this question, some experimental findings concerning socalled "agency distortion" can be used; experimental manipulations to deliberately bring about mismatches between predicted and actual outcomes of bodily actions can result in significant decreases of the sense of agency. ${ }^{50}$ In other words, so long as predicted and actual outcomes properly match each other, the sense of agency increases, even though the subjects are not really in control of the outcomes. ${ }^{51}$ If that is the case, the question about the possibility of keeping the relevant secondary attention to the driving situation without any real voluntary action moves to other questions. They are questions such as how to make drivers' predicted and actual outcomes properly match with each other even without drivers' real control over the vehicle; how, in the first place, to make the drivers keep predicting, through their relevant secondary attention, the outcomes of their bodily movements or movements of the driver-car.

One suggestion is to provide the drivers with a joint control or a "wemode" which enables them to control the car together with the system on an autonomous vehicle to preserve the sense of agency. For that purpose, "the

\footnotetext{
${ }^{48}$ Aron Gurwitsch, The Field of Consciousness: Theme, Thematic Field, and Margin, ed. by R. M. Zaner (Dordrecht: Springer, 2010), 53.

${ }^{49}$ Edmund Husserl, Wahrnehmung und Aufmerksamkeit: Texte aus dem Nachlass (18931912), ed. by Thomas Vongehr and Regula Giuliani, (Dordrecht: Kluwer, 2004), 96-100.

50 Atsushi Sato and Asako Yasuda, "Illusion of Sense of Self-agency: Discrepancy between the Predicted and Actual Sensory Consequences of Actions Modulates the Sense of Selfagency, but Not the Sense of Self-ownership," in Cognition, 94 (2005).

${ }^{51}$ Daniel M. Wegner and Thalia Wheatley, "Apparent Mental Causation: Sources of the Experience of Will," in American Psychologist, 54 (1999).
} 


\section{MOBILITY TECHNOLOGIES}

system should not take over all the control from a driver," but merely "detect the driver's intention and assist in its fulfillment without disturbing the sense of agency." 52 This suggestion aims to restrict the system's role, which seems more or less unrealistic considering current developments of autonomous vehicles.

Another suggestion to solve this problem is to provide the drivers "with different types of information such as a status update and a suggested course of action" to increase situational awareness, namely, "the perception and comprehension of information that allows an individual to project future courses of action needed to respond to a dynamic environment." 53 This suggestion appears, initially at least, to be more promising than the first one. However, it is difficult to tell as to what extent this suggestion is consistent with the autonomous vehicle's promised advantages. For example, driving in general provides the drivers "with a unique opportunity to think about things other than driving." 54 Cars are not only "a prosthesis of the human body" but also "a prosthesis of the human mind," which allows drivers to engage in secondary or driving-irrelevant tasks such as free-floating or deliberative "everyday cognitive journeys" or "mind travels." 55 Autonomous vehicles can extend and enrich such experiences by granting drivers more disengagement from the duties of driving. It is questionable whether providing the driver with more information can avoid conflict with such promises.

Another suggestion is to reinforce user design that serves to keep the driver's sense of agency. ${ }^{56}$ The measure to reinforce user design may be a placebo effect employing, for instance, "placebo buttons" or a "truly functionless steering wheel." 57 Albeit this kind of attention-grabbing suggestion is technically feasible, the question remains of how much it is consistent with the promised advantages of the autonomous vehicle.

Heretofore, no satisfactory solution seems possible to keep the driver's sense of agency at Level 3 for safety reasons unless this level is altogether renounced to skip to Level 4 or 5, which is, as aforementioned, what some carmakers are doing. This paper, committed to speculative rather

\footnotetext{
52 Wen, Kuroki, and Asama, "Sense of Agency in Driving Automation," 9.

${ }^{53}$ Luke Petersen, Lionel Robert, X. Jessie Yang, and Dawn M. Tilbury, "Situational Awareness, Driver's Trust in Automated Driving Systems and Secondary Task Performance," in SAE International Journal of Connected and Autonomous Vehicles, Forthcoming (2019), 4.

${ }^{54}$ Lynne Pearce, Drivetime: Literary Excursions in Automotive Consciousness (Edinburgh: Edinburgh University Press: 2016), ix.

${ }^{55} \mathrm{Ibid} ., 1$.

${ }^{56}$ See James W. Moore, "What Is the Sense of Agency and Why Does It Matter?," in Frontiers in Psychology, 7 (2016), 6-7.

${ }^{57}$ Tim Hwang, "Back Stage at the Machine Theater - A Look at the Theatrics Guiding User Interaction," in Medium (11 April 2015), <https://medium.com/re-form/back-stage-at-themachine-theater-530f973db8d2>.

(c) 2021 Taehee Kim

https://www.kritike.org/journal/special issue 2021/kim april2021.pdf

ISSN 1908-7330

(cc) BY-NC-ND
} 
than technical discussions, cannot investigate how to materialize the possible technical solutions. However, some further discussion might be useful to cast light upon this urgent problem, drawing on Husserl's insights into how it might be possible for a passenger to keep a kinesthetic sense and a sense of agency, as quoted below.

If I am seated in the train car, however, then "I move with it," but without kinaesthetic change. In the first place, stationary kinaesthetic states, connected to [the car's] stationary images, motivate stationary Objects. Here I have a stationary kinaesthetic state connected to moving images of the surroundings and to stationary images of the car and of my Body. Yet this does precisely not mean that the surroundings move but, on the contrary, that they are stationary. And it does not mean that I am stationary but that I move (am moved). Should we say that the car, with its states of movement, or with the corresponding phenomenological changes, assumes the function of kinaesthetic sensation?58

When passengers are seated and traveling in the train car (Wagen), the kinesthetic state regarding their body in a proper sense is no doubt stationary. Still, based on the fact that images of the moving surroundings undergo certain phenomenological changes corresponding to the car's movement, Husserl claims the possibility for the car to assume the function of kinesthesis. Notwithstanding the interrogative sentence at the end of the quotation reveals certain hesitation, Husserl argues elsewhere that "I am appropriated to the vehicle." "Equipment in the most original sense" is "physical objects, with which both the most original and immediate bodily action and the next mediate bodily action affecting the external things gain the new form." This new form, "incorporating a thing which extends the body, can more effectively and purposefully affect the external things." Among the various kinds of equipment (Zeug), the vehicle (Fahr-zeug) is "unified with the whole body" in contrast to a tool (Handwerk-zeug) unified only with a part of my body. Thus, "non-bodily things" such as the vehicle "are not kinesthetic limbs and do not possess own kinesthesis but do in a sense take part in the kinesthesis," so that "now the body itself can be considered equivalent to the non-bodily things." 59

\footnotetext{
58 Edmund Husserl, Thing and Space: Lectures of 1907, trans. by R. Rojcewicz (Dordrecht; Boston: Kluwer Academic Publishers, 1997), 243.

59 Edmund Husserl, Zur Phänomenologie der Intersubjektivität. Texte aus dem Nachlass. Dritter Teil: 1929-1935, ed. by Iso Kern (Den Haag: Nijhoff, 1973), 276-277. (My own translation.)
} 


\section{MOBILITY TECHNOLOGIES}

Of course, this statement of Husserl seems to be contrary to his original account of kinesthesis, suggesting that we become mere passengers without any sense of agency once this kinesthesis is entirely removed. At the same time, it seems that even a mere passenger actually preserves some sense of agency. It happens now and then that if a traffic light turns red and the driver does not slow down for whatever reason, the passenger in the front seat urgently intervenes to warn them to stop the car. Paying attention to and participating in the situation somehow, they are continually anticipating both the car's movement and the intentional feedback corresponding to this movement. Then, not actually executing their kinesthesis at all, this passenger could be said to still preserve their agency to some extent based on the past kinesthetic experiences pertinent to these situations.

Indeed, despite Husserl's more or less speculative statement about riding in a train, it is not ultimately settled whether and how the mere passenger of a vehicle without the potential to intervene directly in the movement of the car could still constitute the driver-car hybrid. Some relevant qualitative research or scientific experiments in psychology and cognitive science, for instance, should be conducted for such thoughtexperiments to be confirmed or dismissed. Notwithstanding the general difficulties involved in this problem, we could understand such "hybrid assemblages" at least at Level 3 as assuming and even facilitating the "kinaesthetic sense" by extending "human capacities into and across the external world." 60

Concerning whether and how mere occupants of an autonomous vehicle may preserve the attention on the situation, it is worth considering the phenomenological reflection on conventional driving by Lynne Pearce. Even the drivers of a conventional car do not pay full attention to the road but "think - and converse-about non-driving-related matters, as well as perform secondary manual tasks, without necessarily compromising our safety." 61 These phenomena could amount even to daydreaming because the "supervisory attention system" is still ready to "spring into action," if needed. ${ }^{62}$ In this context, it is interesting whether this ability is available only for experienced drivers or even for novice drivers or even persons without a driving license in an autonomous vehicle. Putting aside this question, the answer of which may require further empirical research, though, this "ability to pursue 'secondary tasks' while driving" 63 is undoubtedly an illuminating analogy for the driver or occupant in an autonomous vehicle at Level 3.

${ }^{60}$ Urry, Mobilities, 48

${ }^{61}$ Pearce, Drivetime, 162.

62 Ibid., 164.

${ }^{63} \mathrm{Ibid}$.

(c) 2021 Taehee Kim

https://www.kritike.org/journal/special issue 2021/kim april2021.pdf

ISSN 1908-7330

(c) $\mathrm{BY}-\mathrm{NC}-\mathrm{ND}$ 
Correspondingly, even as an occupant or a passenger on an autonomous vehicle at Level 3, we can still possess a sort of kinesthesis and sense of agency by being unified with the car, which assumes the kinesthetic function. The car as driver's extended body or the driver-car is "neither a thing nor a person; it is an assembled social being that takes on properties of both and cannot exist without both." 64 Resorting to the "distinctive ontology" that is generated by the "a metaphysical merger, an intertwining of the identities of the driver and a car [...] in the form of a person-thing, a humanized car or, alternatively, an automobilized person," 65 is needed; how the driver's stable attention in this driver-car assemblage is to materialize remains a research subject.

\section{Concluding Remarks}

The automobility system as "the predominant global form of 'quasiprivate' mobility that subordinates other mobilities of walking, cycling, travelling by rail and so on"66 is approaching an unprecedented breakthrough; at the core of which autonomous vehicles will be organized into a whole networked system of mobilities. This paper addressed this subject matter in terms of an issue closely related to the legal and ethical debates on the responsibility of autonomous driving, the driver's sense of agency. From a phenomenological point of view, this paper dealt with this issue from the perspective of everyday bodily movement, manual driving, and autonomous driving, focusing on Level 3 of driving automation to analyze the problems that can arise in the transfer of control from system to the human driver. The question was how the human driver, not being fully engaged in driving tasks, can still attend to the driving situation to intervene and take control back, if needed. As a philosophical speculation, this paper, far from being committed to offering any concrete technical suggestions, has suggested an ontological possibility to consider the driver-car assemblage as an agent that pays relevant secondary attention to and is responsible for the driving situation.

An obvious desideratum of this speculation is that, being a phenomenological approach in methodology, it is not a qualitative approach per se. In this respect, it is hoped that this paper's results be tested and

${ }^{64}$ Tim Dant, "The Driver-Car," in Theory, Culture \& Society, 21 (2004). Not only for driving in itself, but also "in their interactions, drivers merge with their cars" which means that they "seek intelligibility mainly from the external automobile body." Büscher, Coulton, Efstratiou, Gellersen, and Hemment, "Connected, Computed, Collective: Smart Mobilities," 139.

${ }^{65}$ Jack Katz, How Emotions Work (Chicago: University of Chicago Press, 2000), 33 (as cited in Sheller, "Automotive Emotions," 228).

${ }^{66}$ John Urry, "The 'System' of Automobility," in Theory, Culture \& Society, 21 (2004), 26.

(C) 2021 Taehee Kim

https://www.kritike.org/journal/special issue 2021/kim april2021.pdf

ISSN 1908-7330

(cc) BY-NC-ND 


\section{MOBILITY TECHNOLOGIES}

supplemented with concrete qualitative studies targeting the drivers or the users of autonomous vehicles. Furthermore, while taking for granted that the sense of agency is closely linked to the issue of legal and ethical responsibilities of autonomous vehicle's drivers, this paper is not engaged in an in-depth discussion on what this link could be like, primarily through the conscious and enacted freedom as a medium between them. Correspondingly, this paper has not directly addressed these normative debates of freedom and responsibility pertaining to autonomous vehicles' development, such as whether or to what extent it is desirable to develop autonomous vehicles, making redundant human freedom and responsibility. While this paper is primarily directed to the scholars working on agency from the philosophical and psychological perspective, it seems meaningful at the end of this discussion to refer to the overall context of this discussion from the normative perspective.

Technologies have induced fundamental and irrevocable changes in the human condition. In particular, by drastically changing forms, scales, and paces regarding various movements of people, things, and information, and so on, innovations in mobility technologies have altered the power-geometry of cities and spaces. Above all, "mobility artificial intelligence" embodied in autonomous vehicles is being connected to the whole mobility infrastructure to the extent that "trains of driverless cars allow the meeting of wills to be replaced by calculations of logistics." ${ }^{67}$ In the long term, this kind of technology will be "invisible in fact as well as in metaphor," 68 making such intelligent systems "so imbedded, so fitting, so natural, that we use [them] without even thinking about it." 69 Such technologies transform human beings' lived experiences of the world, that is, the relationship between human beings and the world. Even though technologies not only extend human sensations but also reduce them, ${ }^{70}$ the discussions on cutting-edge technologies regrettably overlook these aspects of sensory reduction. In this respect, there is a need to properly understand potential problems caused by the (current) inability of intelligent systems such as autonomous vehicles "to annotate and categorize the driving environment in terms of human values and moral understanding." 71

\footnotetext{
${ }^{67}$ Laurier and Dant, "What We Do Whilst Driving," 241.

${ }^{68}$ Mark Weiser, "The Computer for the 21st Century," in Scientific American, 265 (1991), 95.

${ }^{69}$ Mark Weiser, "Creating the Invisible Interface," in Proceedings of the 7th Annual ACM Symposium on User Interface Software and Technology, ed. by Pedro Szekely (New York: ACM Press: 1994).

${ }^{70}$ Ihde, Technics and Praxis, 9.

${ }^{71}$ Cunneen, Mullins, and Murphy, "Autonomous Vehicles and Embedded Artificial Intelligence," 706.

(c) 2021 Taehee Kim

https://www.kritike.org/journal/special issue 2021/kim april2021.pdf

ISSN 1908-7330
}

(c) ) BY-NC-ND 
Considered from a historical point of view, these potential problems are well demonstrated; the neologism of "automobile" was meant originally to refer to a new machine "that moves autonomously but simultaneously enables the driver to move autonomously." 72 Nevertheless, due to the "impossibilities of automobility," this ideal of the autonomy of human drivers has been disenchanted; "the more cars are around, the more rules have to be invented" "to allow the regime of automobility to work 'normally,' even though this 'normality' might be contradictory to the image of a completely autonomous movement."73 Adding the qualification "autonomous" to the "automobile" that was already meant to be autonomous, the same ideological phrase seems to recur; it is questionable whether "autonomous automobiles" enable not only themselves but also human beings to be more autonomous. Granted, "cars would become less like horseless carriages and much more like the carriages of a train, occupied only by passengers," 74 questions not only from a legal and ethical viewpoint but from a safety standpoint arise. Whether and to what extent is it desirable to remove the drivers' sense of agency and responsibility? Whether and to what extent is it desirable to make autonomous vehicles at Levels 4 or 5 , which rarely involve human drivers, come into being? These questions, which are by and large lacking in recent discussions about new mobility technologies, are especially crucial given superficially optimistic or inordinately enthusiastic predictions that "are ideologically driven, especially when coming from actors with deep investments in such future." 75 In this respect, the ideological aspects figuring in imaginations of the autonomous vehicle that "reconfigure and reproduce the historically gendered and raced representations, meanings, and practices of (auto)mobility" also needs to be examined to reimagine the future of (auto)mobility. ${ }^{76}$

The Academy of Mobility Humanities, Konkuk University, South Korea

${ }^{72}$ Matthew Paterson, "Carbon," in The Routledge Handbook of Mobilities, ed. by Peter Adey, David Bissell, Kevin Hannam, Peter Merriman, and Mimi Sheller (London: Routledge: 2014), 328-329.

73 Steffen Böhm, Campbell Jones, Chris Land, and Mat Paterson, "Introduction: Impossibilities of Automobility," in Against Automobility, ed. by Steffen Böhm, Campbell Jones, Chris Land, and Mat Paterson (Oxford: Blackwell, 2006), 11.

${ }^{74}$ Laurier and Dant, "What We Do Whilst Driving," 223.

${ }^{75}$ Mimi Sheller, Mobility Justice: The Politics of Movement in an Age of Extremes (New York: Verso, 2018), 71.

76 Julia M. Hildebrand and Mimi Sheller, "Media Ecologies of Autonomous Automobility: Gendered and Racial Dimensions of Future Concept Cars," Transfers, 8 (2018), 64.

(C) 2021 Taehee Kim

https://www.kritike.org/journal/special issue 2021/kim april2021.pdf

ISSN 1908-7330

(cc) BY-NC-ND 


\section{MOBILITY TECHNOLOGIES}

\section{References}

Armstrong, David M., "The Nature of Mind," in Arts: The Proceedings of the Sydney University Arts Association, 3 (1966).

Bayne, Tim, "The Phenomenology of Agency," in Philosophy Compass, 3 (2008).

Berberian, Bruno, Jean-Christophe Sarrazin, Patrick Le Blaye, and Patrick Haggard, "Automation Technology and Sense of Control: A Window on Human Agency," in PLOS One, 7 (2012).

Böhm, Steffen, Campbell Jones, Chris Land, and Mat Paterson, "Introduction: Impossibilities of Automobility," in Against Automobility, ed. by Steffen Böhm, Campbell Jones, Christ Land, and Mat Paterson (Oxford: Blackwell, 2006).

Büscher, Monika, Paul Coulton, Christos Efstratiou, Hans Gellersen, and Drew Hemment, "Connected, Computed, Collective: Smart Mobilities," in Mobilities: New Perspectives on Transport and Society, ed. by Margaret Grieco and John Urry (London: Routledge, 2015).

Coeckelbergh, Mark, "Responsibility and the Moral Phenomenology of Using Self-driving Cars," in Applied Artificial Intelligence, 30 (2016).

Cunneen, Martin, Martin Mullins, and Finbarr Murphy, "Autonomous Vehicles and Embedded Artificial Intelligence: The Challenges of Framing Machine Driving Decisions," in Applied Artificial Intelligence, 33 (2019).

Dant, Tim, "The Driver-Car," in Theory, Culture \& Society, 21 (2004).

, "Drivers and Passengers," in The Routledge Handbook of Mobilities, ed. by Peter Adey, David Bissell, Kevin Hannam, Peter Merriman, and Mimi Sheller (London: Routledge: 2014).

Dreyfus, Hubert L., Skillful Coping: Essays on the Phenomenology of Everyday Perception and Action, ed. by Mark Wrathall (Oxford: Oxford University Press, 2014).

Dreyfus, Stuart E. and Hubert L. Dreyfus, A Five-Stage Model of the Mental Activities Involved in Directed Skill Acquisition (Berkeley: California University Berkeley Operations Research Center, 1980).

Frith, Chris D., "Action, Agency and Responsibility," in Neuropsychologia, 55 (2014).

Gallagher, Shaun and Dan Zahavi, The Phenomenological Mind: An Introduction to Philosophy of Mind and Cognitive Science (New York: Routledge, 2008).

Gurwitsch, Aron, The Field of Consciousness: Theme, Thematic Field, and Margin, ed. by R. M. Zaner (Dordrecht: Springer, 2010).

(c) 2021 Taehee Kim

https://www.kritike.org/journal/special issue 2021/kim april2021.pdf

ISSN 1908-7330

(c) $)$ BY-NC-ND 
Haggard, Patrick and Manos Tsakiris, "The Experience of Agency: Feelings, Judgments, and Responsibility," in Current Directions in Psychological Science, 18 (2009).

Henschke, Adam, "Trust and Resilient Autonomous Driving Systems," in Ethics and Information Technology, 22 (2020).

Hildebrand, Julia M. and Mimi Sheller, "Media Ecologies of Autonomous Automobility: Gendered and Racial Dimensions of Future Concept Cars," in Transfers, 8 (2018).

Husserl, Edmund, Zur Phänomenologie der Intersubjektivität. Texte aus dem Nachlass. Dritter Teil: 1929-1935, ed. by Iso Kern (Den Haag: Nijhoff, 1973).

Ideas Pertaining to a Pure Phenomenology and to a Phenomenological Philosophy, Book II, trans. by R. Rojcewicz and A. Schuwer (Boston: Kluwer Academic Publishers, 1989).

Thing and Space: Lectures of 1907, trans. by R. Rojcewicz (Dordrecht; Boston: Kluwer Academic Publishers, 1997).

Wahrnehmung und Aufmerksamkeit: Texte aus dem Nachlass (18931912), ed. by Thomas Vongehr and Regula Giuliani (Dordrecht: Kluwer, 2004).

Hwang, Tim, "Back Stage at the Machine Theater - A Look at the Theatrics Guiding User Interaction," in Medium (11 April 2015), $<$ https://medium.com/re-form/back-stage-at-the-machine-theater$530 \mathrm{f} 973 \mathrm{db} 8 \mathrm{~d} 2>$.

Ihde, Don, "The Experience of Technology: Human-Machine Relations," in Cultural Hermeneutics, 2 (1974).

Technology and the Lifeworld: From Garden to Earth (Bloomington: Indiana University Press, 1990).

Technics and Praxis: A Philosophy of Technology (Dordrecht: Reidel, 1979).

Kim, Jooyoung, Taehee Kim, Jinhyoung Lee, and Inseop Shin, “Exploring Humanistic Layers of Urban Travel: Representation, Imagination, and Speculation," in Transfers, 9 (2019).

Kim, Taehee, "Significance of Kinaesthesis for Mobility Humanities: A Phenomenological Contribution to the New Mobilities Paradigm," in Universitas-Monthly Review of Philosophy and Culture, 47 (2020).

Labastin, Benjiemen A., "A Search for a Model of Critical Engagement with Technology: Feenberg's Instrumentalization Theory or MASIPAG's Struggle against Corporate Control of Agricultural Technologies?," in KRITIKE: An Online Journal of Philosophy, 13 (2019).

Laurier, Eric and Tim Dant, "What We Do Whilst Driving: Towards the Driverless Car," in Mobilities: New Perspectives on Transport and Society, ed. by Margaret Grieco and John Urry (London: Routledge, 2015).

(C) 2021 Taehee Kim

https://www.kritike.org/journal/special issue 2021/kim april2021.pdf

ISSN 1908-7330

(cc) BY-NC-ND 


\section{MOBILITY TECHNOLOGIES}

Lenz, Barbara and Eva Fraedrich, "New Mobility Concepts and Autonomous Driving: The Potential for Change," in Autonomous Driving: Technical, Legal and Social Aspects, ed. by Markus Mauer, Chris Gerdes, Barbara Lenz, and Hermann Winner (Cham: Springer Nature, 2016).

Lin, Patrick, "Why Ethics Matters for Autonomous Cars," in Autonomous Driving: Technical, Legal and Social Aspects, ed. by Markus Mauer, Chris Gerdes, Barbara Lenz, and Hermann Winner (Cham: Springer Nature, 2016).

Martines, Michael, "Ford Rethinks Level 3 Autonomy," in Automotive News Europe (20 January 2019), $<$ https://europe.autonews.com/automakers/ford-rethinks-level-3autonomy $>$.

Merleau-Ponty, Maurice, Phenomenology of Perception (London: Routledge, 1962).

Merriman, Peter and Lynne Pearce, "Mobility and the Humanities," in Mobility and the Humanities, ed. by Peter Merriman and Lynne Pearce (London: Routledge, 2018).

Mitchell, Russ, "When Robots and Humans Take Turns at the Wheel," in Los Angeles Times (22 September 2016), $<$ https://www.latimes.com/business/autos/la-fi-hy-driverless-levelstesla-ford-gm-mercedes-volvo-google-20160922-snap-story.html>.

Moore, James W., "What Is the Sense of Agency and Why Does It Matter?" in Frontiers in Psychology, 7 (2016).

Øvergård, Kjell Ivar, "A Video-Based Phenomenological Method for Evaluation of Driving Experience in Staged or Simulated Environments," in Embodied Minds - Technical Environments: Conceptual Tools for Analysis, Design and Training, ed. by Thomas Hoff and Cato Alexander Bjørkli (Trondheim: Tapir Academic Press, 2008).

Paterson, Matthew, "Carbon," in The Routledge Handbook of Mobilities, ed. by Peter Adey, David Bissell, Kevin Hannam, Peter Merriman, and Mimi Sheller (London: Routledge: 2014), 328-329.

Pearce, Lynne, Drivetime: Literary Excursions in Automotive Consciousness (Edinburgh: Edinburgh University Press: 2016).

Petersen, Luke, Lionel Robert, X. Jessie Yang, and Dawn M. Tilbury, "Situational Awareness, Driver's Trust in Automated Driving Systems and Secondary Task Performance," in SAE International Journal of Connected and Autonomous Vehicles, Forthcoming (2019).

SAE International, "SAE International Releases Updated Visual Chart for Its 'Levels of Driving Automation' Standard for Self-Driving Vehicles," in SAE.org (11 December 2018), <https://www.sae.org/news/pressroom/2018/12/sae-international-releases-up dated-visual-chart-for-

(c) 2021 Taehee Kim

https://www.kritike.org/journal/special issue 2021/kim april2021.pdf

ISSN 1908-7330

(cc) BY-NC-ND 
its- $\%$ E2\%80\%9Clevels-of-driving-automation $\%$ E2\%80\%9D-

standard-for-self-driving-vehicles>.

Sato, Atsushi and Asako Yasuda, "Illusion of Sense of Self-agency: Discrepancy between the Predicted and Actual Sensory Consequences of Actions Modulates the Sense of Self-agency, but Not the Sense of Self-ownership," in Cognition, 94 (2005).

Sheller, Mimi, "Automotive Emotions: Feeling the Car," in Theory, Culture $\mathcal{E}$ Society, 21 (2004).

Mobility Justice: The Politics of Movement in an Age of Extremes (Brooklyn, NY: Verso, 2018).

Sheller, Mimi and John Urry, "The New Mobilities Paradigm," in Environment and Planning A, 38 (2006).

Urry, John, "The 'System' of Automobility," in Theory, Culture \& Society, 21 (2004).

Mobilities (Cambridge: Polity Press, 2007).

Wegner, Daniel M. and Thalia Wheatley, "Apparent Mental Causation: Sources of the Experience of Will," in American Psychologist, 54 (1999).

Weiser, Mark, "The Computer for the 21st Century," in Scientific American, 265 (1991).

"Creating the Invisible Interface," in Proceedings of the 7th Annual ACM Symposium on User Interface Software and Technology, ed. by Pedro Szekely (New York: ACM Press, 1994).

Wen, Wen, Yoshihiro Kuroki, Hajime Asama, "The Sense of Agency in Driving Automation," in Frontiers in Psychology, 10 (2019).

Williams, Bernard, Ethics and the Limits of Philosophy (London: Routledge, 2011).

Wilson, Margaret, "Six Views of Embodied Cognition," in Psychonomic Bulletin \& Review, 9 (2002).

Wolf, Ingo, "The Interaction Between Humans and Autonomous Agents," in Autonomous Driving: Technical, Legal and Social Aspects, ed. by Markus Mauer, Chris Gerdes, Barbara Lenz, and Hermann Winner (Cham: Springer Nature, 2016).

(C) 2021 Taehee Kim

https://www.kritike.org/journal/special issue 2021/kim april2021.pdf

ISSN 1908-7330 